\title{
Palm Print Confirmation Utilizing Contourlet
}

\author{
B.Sharmila \\ Assistant Professor \\ Department of Computer Science \& Engineering \\ Vignan Institute of Technology \& Science, Hyderabad
}

\begin{abstract}
Biometric processing offers a compelling way to deal with recognize individual personality by utilizing person's one of a kind, solid and stable physical or conduct attributes. This task portrays another strategy to confirm people dependent on palmprint recognizable proof. In this work the utilization of palm print for the individual distinguishing proof should be possible by utilizing the contourlet changes. The palm print confirmation framework comprises of I) Extracting the picture from the database .ii) Preprocessing iii) Feature Extraction utilizing contourlet transforms. iv) Evaluation of results. The contourlet change is a multidirectional and multiscale change that developed by the mix of LP (Laplacian pyramid) and DFB (directional channel bank). It can successfully catch smooth shapes that are the predominant aspects in palm print pictures. Euclidean separation is utilized as classifier in this proposed framework. Analyses are led on poly $u$ palm print database. 625 palm prints acquired from 125 clients with 5 examples have been gathered and 375 pictures are given for preparing and 625 pictures have been given for testing and assessed for the presentation of the proposed framework.
\end{abstract}

\section{INTRODUCTION}

PC Vision is the science and innovation of machines that see. As a logical control, PC vision is worried about the hypothesis for building fake frameworks that get data from pictures. The picture information can take numerous structures, for example, a video arrangement, sees from various cameras, or multi-dimensional information from a clinical scanner.

$\mathrm{PC}$ vision can likewise be portrayed as a supplement (however not really the inverse) of organic vision. In organic vision, the visual impression of people and different creatures are considered, bringing about models of how these frameworks work regarding physiological procedures. PC vision, then again, examines and portrays counterfeit vision framework that is actualized in programming as well as equipment. Interdisciplinary trade among organic and PC vision has demonstrated progressively productive for the two fields. Sub-areas of PC vision incorporate scene recreation, occasion identification, following, object acknowledgment, getting the hang of, ordering, movement estimation, and picture rebuilding.

\section{$>$ Biometrics}

A biometric framework gives programmed acknowledgment of an individual dependent on a one of a kind element or trademark controlled by the person. Biometric frameworks have been created dependent on fingerprints, facial highlights, voice, hand geometry, penmanship, and the retina.

Biometric acknowledgment can be utilized in Identification mode, where the biometric framework recognizes an individual from the whole enlisted populace via looking a database for a match dependent on the biometric. For instance, a whole database can be looked to confirm an individual has not applied for privilege benefits under two distinct names. This is now and then called "oneto-many" coordinating. A framework can likewise be utilized in Verification mode, where the biometric framework confirms an individual's guaranteed character from their recently selected example. This is additionally called "balanced" coordinating. In most PC access or system get to situations, confirmation mode would be utilized. A client enters a record, client name, or embeds a token, for example, a savvy card, yet as opposed to entering a secret key, a basic touch with a finger or a look at a camera is sufficient to verify the client.

\section{$>$ Palm print recognition}

Palm print is viewed as one of the most one of a kind, solid and stable individual qualities and palm print confirmation gives a ground-breaking intends to validate people for some security frameworks. There has been a popularity for individual ID and confirmation for security reasons. Biometrics is worried about the remarkable, dependable and stable individual physiological attributes, for example, fingerprints, palm print, facial highlights, iris example, retina and hand geometry, or some part of conduct, similar to discourse and penmanship. As of late, issues on face acknowledgment and iris-based confirmation have been concentrated broadly, which brings about effective advancement of biometric frameworks for business applications. The basis to pick hand includes as a base for character check is begun by its ease of use, condition Flexibility and separating capacity. Subsequently, it is basic to build up a viable way to deal with programmed palm print ID and check for security reasons. 


\section{$>$ Applications of palm print recognition}

The worries on protections particularly at significant and significant spots like air terminals, ports, customs, jails, outskirt posts, government structures, private structures and clubs, banks, high-security research facilities and structures, atomic force plants, military offices and locales, key destinations, open zone and so on has made popularity of a framework equipped for dealing with any danger from any sort of sources. Fingerprinting expects one to put his/her hand/palm/finger on a machine and afterward the machine peruses the data and stores it on a database. It peruses edge from various pieces of the hand extending from fingers, designs and even toes. The adaptability of rubbing edge skin implies that no two finger or palm prints are ever precisely the equivalent.

\section{LITERATURE SURVEY}

The creators G.Y.Chen and B.Kegl, [1] proposed another palm print characterization by utilizing the contourlet highlights. The contourlet change has the multiscale and time recurrence confinement properties of wavelets, yet additionally offers a high level of directionality and anisotropy. AdaBoost is utilized as a classifier in the trial. It is the expansion of wavelet change in $2 \mathrm{D}$ by utilizing non-divisible and DFB to beat the impediments of wavelets. It depends on a proficient two dimensional multiscale and directional channel bank that can manage pictures having smooth forms.

Drawbacks: The characterization rate is low.

The creators G.Y.Chen, T.D.Bui and A.Krzyzak,[2] managed the palm print characterization is finished by utilizing double tree complex wavelet change. The double tree complex wavelet change has such significant properties as the inexact move invariance and high directional selectivity .bolster vector machines are utilized as a classifier and the Gaussian spiral premise work bit is chosen in this investigation. The grouping rate is $97 \%$ by utilizing double tree complex wavelet change.

Drawbacks: The double tree complex wavelet produces higher order rate than the scalar wavelet i.e., 97\% as it were. The double tree complex wavelet highlights ought to be utilized for invariant palm print grouping rather than the scalar wavelet highlights.

The creators M.N.Do and M.Vetterli, [3] managed directional multiresolution examination that gives progressive refinements at both spatial and directional goals. They previously distinguished two key highlights of another picture portrayal that improves over the distinct 2-D wavelet change, in particular directionality and anisotropy which is a discrete change that gives an inadequate extension to run of the mill pictures having smooth forms. They built up another channel bank structure, the contourlet channel bank that can give an adaptable multiscale and directional deterioration for picture.
Drawbacks: Used just two key highlights (i.e., directionality and anisotropy)

\section{IMPLEMENTATION}

\section{$>$ Extract the input image:}

This is the primary module in the framework. To start working with the framework first the info picture is to be chosen. This database contains number of individual pictures that are taken under changing lighting, direction and posture. And all the pictures in the database are of a similar size. Examinations are directed on poly u palm print database. 625 palm prints got from 125 clients with 5 examples have been gathered and 375 pictures are given for preparing and 400 pictures are given for testing from the database one picture is chosen and the various tasks are performed on this info picture.

\section{$>$ Image normalization:}

In picture handling, standardization is a procedure that changes the scope of pixel power esteems. Applications incorporate photos with poor difference because of glare, for instance. Standardization is once in a while called differentiate extending. In increasingly broad fields of information handling, for example, computerized signal preparing it is alluded to as unique range development. The reason for dynamic range development in the different applications is ordinarily to bring the picture, or other sort of sign, into a range that is progressively recognizable or typical to the faculties, subsequently the term standardization. Regularly, the inspiration is to accomplish consistency in unique range for a lot of information, signals, or pictures to maintain a strategic distance from mental interruption or weakness. For instance, a paper will endeavor to make the entirety of the pictures in an issue share a comparable scope of grayscale.

Standardization is a straight procedure. On the off chance that the force scope of the picture is 50 to 180 and the ideal range is 0 to 255 the procedure involves taking away 50 from every one of pixel power, making the range 0 to 130 . At that point every pixel power is duplicated by $255 / 130$, making the range 0 to 255 . Auto-standardization in picture handling programming commonly standardizes to the full unique scope of the number framework determined in the picture document design .The standardization procedure will deliver iris districts, which have a similar steady measurements, with the goal that two photograph diagrams of a similar iris under various conditions will have trademark highlights at the equivalent spatial area.

The point of the palm print preprocessing step is to standardize the database, with the goal that a hearty element extraction can be accomplished. Contingent upon the application, palm preprocessing incorporates: Alignment (interpretation, revolution, scaling) and light standardization/relationship. The palm preprocessing step targets normalizing, for example diminishing the blunders that are happened during catch of the pictures. Palm prints are antagonistically influenced by varieties in brightening. In the preprocessing revolution of the palm print for the 
ISSN No:-2456-2165

even arrangement is performed. After the flat arrangement of the palm print morphological activity of expansion is performed to evacuate the gaps in the paired picture. The picture is supplemented lastly separation change is utilized with the chessboard metric to compute the focal point of the palm print. Subsequent to knowing the focal point of the palm print the picture is edited.

\section{Feature Extraction:}

Highlight extraction assumes a significant job in picture ID and confirmation. There are numerous highlights displayed in a palm. There are three chief lines brought about by Flexing hand and wrist in the palm, which are named as heart line, head line and life line, the design of a palm, where a palm is partitioned into three locales, to be specific finger-root district I, inside area II and outside district III. The three stamped speak to the three chief lines (heart line, head line and life line), individually. The two endpoints are dictated by the crossing points of life line and heart line on the two sides of a palm. Because of the strength of the chief lines, the areas of endpoints and their midpoint in a palm stay unaltered as for turn of the hand and the difference in time. Accordingly, these element lines are viewed as solid and stable highlights to recognize an individual from others.

The component extraction for palm print utilizing contourlet change is:

- The contourlet change is an augmentation to the wavelet change in $2 \mathrm{D}$ by utilizing non distinct and DFB.

- Contourlet changes is developed by two channel banks i.e., LP and DFB.

- It permits any number of DFB disintegration levels to be applied at each Laplacian pyramid level.

The contourlet change is utilized as highlight extraction .In this procedure three level disintegration is performed on palm print pictures. In this proposal a twofold channel bank structure for getting saves extension for run of the mill pictures having smooth shapes. Contourlets have lengthened backings at different scales, headings and viewpoint proportions. This permits contourlets to proficiently estimated a smooth form at numerous goals. In the recurrence area, the contourlet change gives a multiscale and directional deterioration.

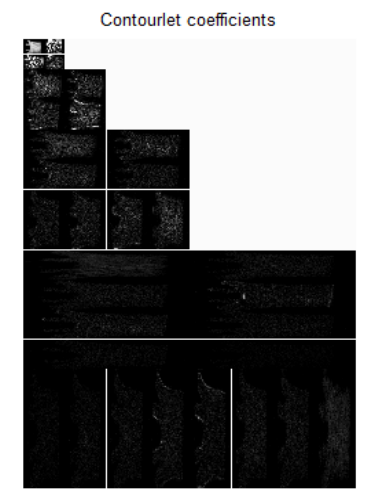

Fig 1:- contourlet transform for palmprint

The LP deterioration at each level produces a down tested lowpass rendition of the first and the contrast between the first and the forecast, bringing about a bandpass image.Decomposition process, where $\mathrm{H}$ and $\mathrm{G}$ are called (lowpass) investigation and blend channels, individually, and $M$ is the inspecting framework. The procedure can be iterated on the coarse (down tested lowpass) signal. This recurrence scrambling occurs in the wavelet channel bank when a highpass channel, after down inspecting, is collapsed once again into the low recurrence band, and along these lines its range is reflected. In the LP, this impact is kept away from by down examining the lowpass channel as it were. The LP utilizing the hypothesis of edges and oversampled channel banks. We demonstrated that the LP with symmetrical channels (that is, the examination and amalgamation channels are time inversion, $\mathrm{h}[\mathrm{n}]=\mathrm{g}[. \mathrm{n}]$, and $\mathrm{g}[\mathrm{n}]$ is symmetrical to it's makes an interpretation of concerning the testing cross section by $\mathrm{M}$ ) furnishes a tight edge with outline limits are equivalent to 1. For this situation, we proposed the utilization of the ideal straight remaking utilizing the double edge administrator.

\section{Classification:}

In science, the Euclidean separation or Euclidean measurement is the "standard" separation between two focuses that one would gauge with a ruler, which can be demonstrated by rehashed utilization of the Pythagorean hypothesis By utilizing this recipe as separation, Euclidean space turns into a measurement space The related standard is known as the Euclidean norm.Older writing alludes to this measurement as Pythagorean measurement. The strategy has been rediscovered various occasions since forever, as it is a coherent expansion of the Pythagorean hypothesis The Euclidean distance between points $\mathrm{P}=\left(\mathrm{p}_{1}\right.$, $\left.\mathrm{p}_{2}, . \mathrm{p}_{\mathrm{n}}\right)$ and $\mathrm{Q}=\left(\mathrm{q}_{1}, \mathrm{q} 2 \ldots \ldots \ldots \mathrm{q}_{\mathrm{n}}\right)$, in Euclidean $\mathrm{n}$-space, is defined as: 


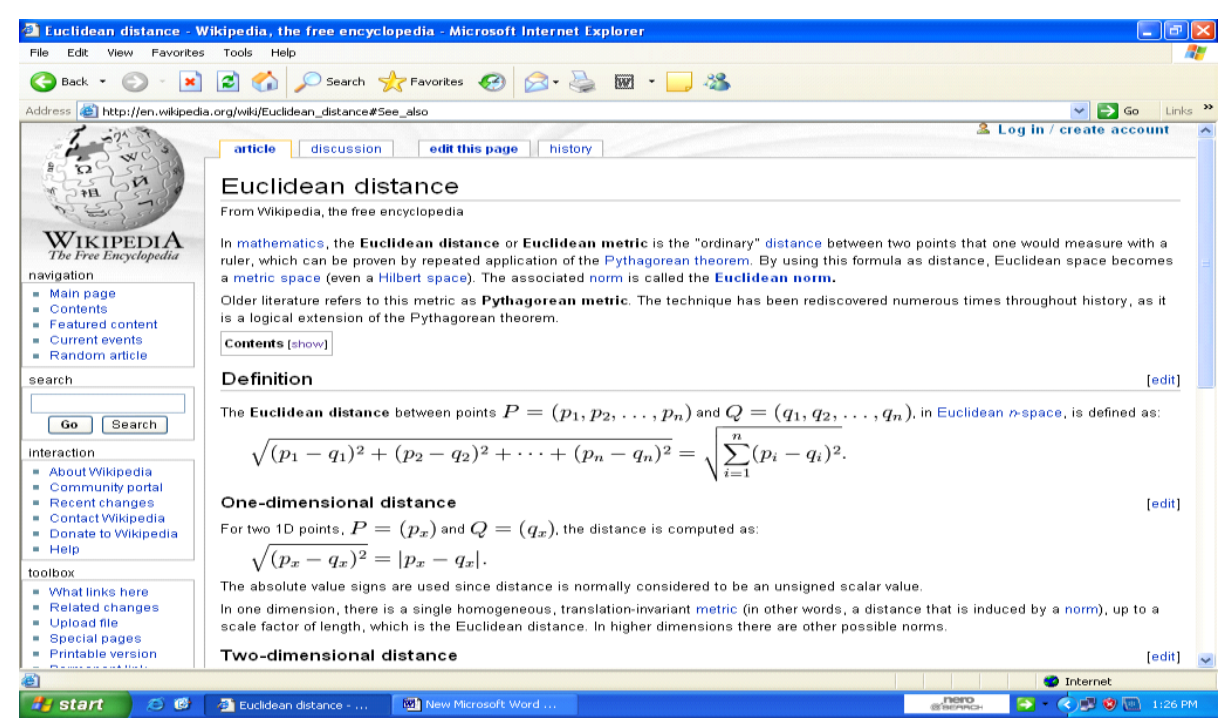

ISSN No:-2456-2165

Fig 2

One-dimensional distance

For two $1 \mathrm{D}$ points, $\mathrm{P}=\left(\mathrm{p}_{\mathrm{x}}, \mathrm{p}_{\mathrm{y}}\right)$ and $\mathrm{Q}=\left(\mathrm{q}_{\mathrm{x}}, \mathrm{q}_{\mathrm{y}}\right)$, the distance is computed as

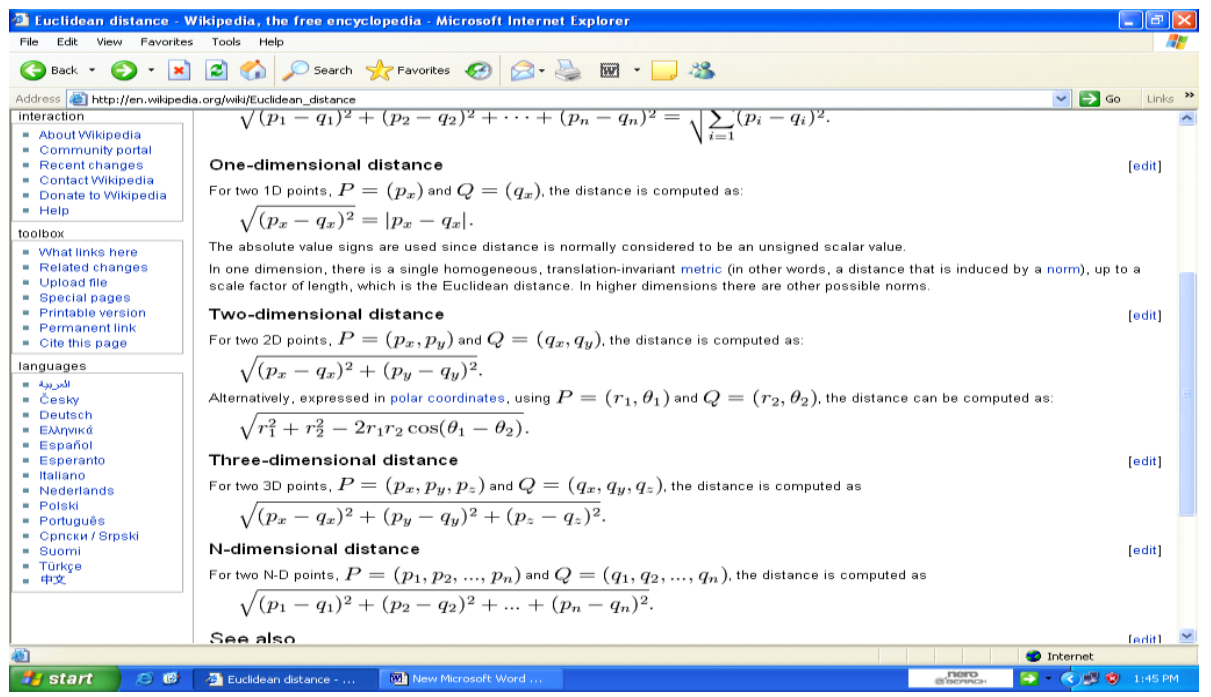

Fig 3

For two 1D points and, the distance is computed as:

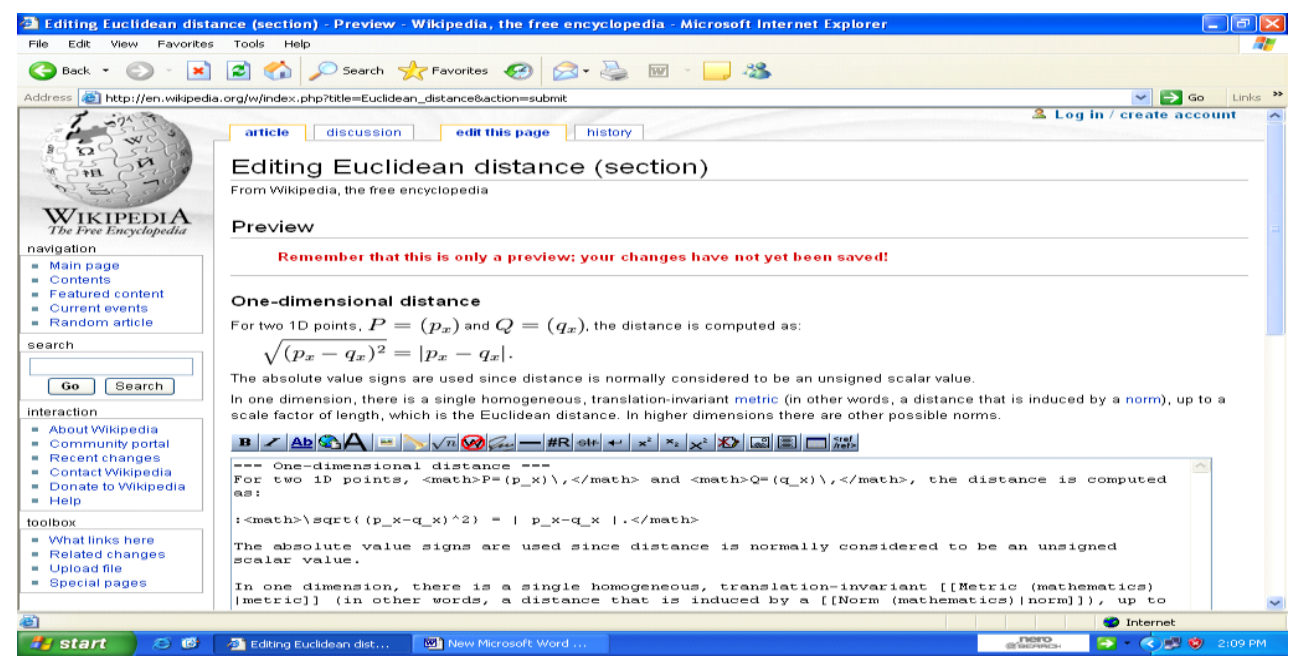

Fig 4 
The total worth signs are utilized since separation is ordinarily viewed as an unsigned scalar value.In one measurement, there is a solitary homogeneous, interpretation invariant measurement (at the end of the day, a separation that is incited by a standard), up to a scale factor of length, which is the Euclidean separation. In higher measurements there are other potential standards. In the order conspire Matching is performed by ascertaining the Euclidean Distance between the info highlight vector and layout include vector. Euclidean separation between two vectors is determined by between two focuses is known as Euclidean separation For $\mathrm{p}(\mathrm{x} 1, \mathrm{y} 1)$ and $\mathrm{q}(\mathrm{x} 2, \mathrm{y} 2)$ the Euclidean separation among $\mathrm{p}$ and $\mathrm{q}$ is characterized as: squaring the distinction between relating components. The separation

$D_{e}(p, q)=\sqrt{ }\left[\left(x_{1}-x_{2}\right)^{2}+\left(y_{1}-y_{2}\right)^{2}\right]$

For the characterization plan to get more precision neural system classifiers like Back-spread calculation, AdaBoost calculation, bolster vector machines can likewise be utilized for order. Along these lines, the precision and the exhibition will be more. In our proposed sytem testing is directed on 625 pictures in the database where 375 are utilized for preparing and 625 are utilized for testing. The results are indicated graphically that the presentation increments by expanding the quantity of preparing mages.

Among the Existing algorithms like SVM retrieval and by directional context modeling our proposed palm print recognition method achieves $99.5 \%$ accuracy.

\section{CONCLUSIONS}

We propose another strategy for palm print grouping by utilizing the contourlet highlights and Euclidean separation. The contourlet change has the multi scale and time recurrence confinement properties of wavelets, yet in addition offers a high level of directionality and anisotropy. These properties are significant in invariant palm print arrangement. Supposedly, this paper is utilized for the contourlet change for invariant palm print acknowledgment.

\section{REFERENCES}

[1]. G.Y.Chen and B.Kegl'Palmprint classification using contourlets", IEEE International Conference on August 2007.

[2]. G.Y.Chen,T.D.Bui and A.Krzyzak"palm print classification using dual tree complex wavelets"IEEE international conference on image processing October 2006.

[3]. M.N.Do and M.Vetterli"'The contourlet transform: an efficient directional multiresolution image representation"IEEE transactions image on processing 2005.

[4]. D. Zhang, W.-K. Kong, J. You and M. Wong, "Online palm print identification," IEEE Transactions on Pattern Analysis and Machine Intelligence, vol. 25, no. 9, pp. 1041-1050, 2003.
[5]. R. Eslami and H. Radha, "Image denoising using translation-invariant contourlet transform," Proc. of IEEE International Conference on Acoustics, Speech, and Signal Processing, Philadelphia, USA, March 18-23, 2005.

[6]. B. Matalon, M. Zibulevsky and M. Elad, "Improved denoising of images using modeling of the redundant contourlet transform," Proc. of the SPIE conference wavelets, Vol. 5914, July 2005.

[7]. J. You, W. Li and D. Zhang, "Hierarchical palmprint identification via multiple feature extraction," Pattern Recognition, vol.35, pp.847-859, 2002.

[8]. Minh.N.Do and Martin Vetterli “contourlets"IEEE transaction, pp.1-27, 2001.

[9]. D. Zhang and W. Shu, "Two novel characteristics in Palmprint verification: Datum point invariance and line feature matching," Pattern Recognition, vol. 32, pp. 691-702, 1999.

[10]. G. Y. Chen and W. F. Xie, "Pattern recognition with SVM and dual tree complex wavelets," Image and Vision Computing, to appear, 2007. 